\title{
Thematic series on Verification and Composition for the Internet of Services and Things
}

\author{
Marco Autili ${ }^{*}$, Dimitra Giannakopoulou ${ }^{2}$ and Massimo Tivoli ${ }^{1}$
}

\begin{abstract}
The Internet of Services and Things is characterized as a distributed computing environment that will be populated by a large number of software services and things. Within this context, software systems will increasingly be built by reusing and composing together software services and things distributed over the Internet. This calls for new integration paradigms and patterns, formal composition theories, integration architectures, as well as flexible and dynamic composition and verification mechanisms. In particular, service- and thing-based systems pose new challenges for software composition and verification techniques, due to changing requirements, emerging behaviors, uncertainty, and dynamicity.
\end{abstract}

\section{Introduction}

This thematic series of the Journal of Internet Services and Applications (JISA) presents a collection of articles around the topic of Verification and Composition for the Internet of Services and Things, including: run-time support for verifying and composing services and things, QoS verification of service- and thing-based systems, crosscutting concerns, and tools, case studies, and use cases.

The Internet of Services and Things promotes a distributed computing environment that will be inhabited by a virtually infinite number of software services and things. Within this context, software systems will increasingly be built by reusing and composing together software services and things distributed over the Internet.

The Future Internet [1] is now a reality, bringing a new distributed computing environment in which the availability of ubiquitous communication is changing people's everyday life. A large number of software services and things are being increasingly available and can be composed to meet user needs. In particular, the Future Internet of Services and the Internet of Things (IoTs) emerge from the convergence of the Future Internet (FI), the Service-Oriented Computing (SOC) paradigm, and the

*Correspondence: marco.autili@univaq.it

'Department of Information Engineering, Computer Science and Mathematics - University of L'Aquila, L'Aquila, Italy

Full list of author information is available at the end of the article
IoT paradigm [2]. Services and things play a central role in this vision as an effective means to achieve interoperability between heterogeneous parties of business processes and IoT networks, and new value-added software systems can be built by reusing and composing services and things.

The Internet of Services and Things is thus radically changing the way software is produced, verified and used, and this calls for new software composition paradigms and patterns, flexible infrastructures and integration architectures, as well as novel modeling and verification methods. Systems are increasingly produced according to a certain goal and by integrating existing components; the focus of system development is on integration of third-party components that are only provided with an interface that exposes the available functionalities and, sometimes, the interaction protocol; after deployment they must be able to cope with dynamically changing requirements and emergent behaviors caused by uncertainty in the surrounding environment. To cope with the changing requirements and emergent behaviors, system designers and software engineers inevitably need to resort to ad-hoc paradigms and technologies to define suitable reconfigurability mechanisms that allow systems to work correctly with respect to the new settlement.

This JISA Thematic Series originates from the Workshop on The Art of Service Composition and Formal Verification for Self-* Systems (VERY*SCART) that was

\section{Springer Open}

(C) The Author(s). 2018 Open Access This article is distributed under the terms of the Creative Commons Attribution 4.0 International License (http://creativecommons.org/licenses/by/4.0/), which permits unrestricted use, distribution, and reproduction in any medium, provided you give appropriate credit to the original author(s) and the source, provide a link to the Creative Commons license, and indicate if changes were made. 
held in York, UK, on September 8, 2015, and which was affiliated with the 13th International Conference on Software Engineering and Formal Methods (SEFM 2015). The event originated from the fusion of the second edition of VERY and the first edition of SCART and was devised for bringing together researchers and practitioners from various areas related to service composition and verification. VERY*SCART 2015 aimed at providing innovative contributions in the research and development of novel Formal Methods (FM) and Software Engineering (SE) approaches to the design, development, validation, and execution of FI applications and self*-systems composed of available components. In particular, the workshop provided the opportunity to discuss how the Future Internet affects the traditional methods and tools, and how facing complexity in terms of scalability, heterogeneity, and dynamicity promotes the integration of FM within SE practices. The goal was to seek answers on how the rigorousness of FM assists engineers while designing, developing, validating, and operating systems that are built via correct-by-construction composition.

Continuing in this direction, this JISA Thematic Series targets new verification and composition techniques able to meet the requirements of modern applications, counteracting the specialization of traditional approaches in order to deal with heterogeneity, dynamicity, adaptation, large scale, mobility, security, etc. We received contributions at various levels: from foundational aspects to concrete application experiments; from modeling to verification and analysis; from componentization to composition; and from deployment to execution.

This Thematic Series was conceived to be highly selective and demanding of high-quality research and technical contributions. We received eight submissions concerning context-aware middleware for IoT, advanced composition techniques for next generation IoT systems, model-driven approaches to the development of IoT system interfaces, context-aware frameworks supporting the dynamic composition of services and things, and data management in IoT. The two out of eight submissions that were selected for publication and appear in this issue are summarized in the following section.

\section{The papers}

The two accepted papers address issues related to the model-driven development of user interfaces for IoT systems via domain-specific components and patterns [3], and a context-aware framework for dynamic composition of process fragments in the Internet of Services [4].

Brambilla et al. [3] propose a model-driven approach to the design of user interfaces of IoT systems, by defining IoT-specific UI components and design patterns. Four research questions are addressed: (i) define the main domain-specific concepts for IoT and the typical use cases; (ii) define a (visual) modeling language for the development of the user interaction aspects of IoT applications; (iii) define a set of design practices that increase productivity and simplifies the design of IoT front-ends; and (iv) implement model-driven tools covering the design, deployment, and execution phases of IoT applications. These research questions concern a number of problems that in the domain of the IoT are worth to be investigated. For instance, the IoT vision is mainly focused on the technological and infrastructure aspect, and on the management and analysis of the huge amount of generated data, while so far the development of front-end and user interfaces for IoT has not played a relevant role in research. On the contrary, user interfaces can play a key role in the acceptance of IoT solutions by final adopters. The paper discusses the requirements and usage scenarios covering the front-end aspects of IoT systems and present a model-driven approach to the design of such interfaces by defining specific components and design patterns using a visual modeling language for IoT applications. An implementation of the proposed solution has been applied to three industrial case studies.

Bucchiarone et al. [4] present a comprehensive framework, and its implementation, for automated service composition that is specifically designed to be used in dynamic execution environments and allows for context-aware service composition and execution. The idea is to organize the composition life cycle in such a way that most human activities can be accomplished at design time. At run time, the composition management, from the synthesis of executable processes to their deployment, is fully automated. Moreover, dynamic changes, e.g., pluggingin a new service into the system, are also accounted for. This is done by exploiting Artificial Intelligence planning techniques that can deal with concrete service models (asynchronous, stateful and nondeterministic services) and allows for rich control- and data-flow requirements. To demonstrate their approach in action and evaluate it, the authors exploited the ASTRO-CAptEvo framework, simulating the operation of a fully automated IoS-based car logistics scenario in the Bremerhaven harbor.

\section{Paper selection process}

There were two independent cycles of submissions and the papers were published as soon as they became ready. Each manuscript went through several revisions before the final acceptance. We invited a number of leading experts in the area to form an editorial committee to throughly review the papers. All manuscripts were reviewed by at least three members of the editorial committee. For each review cycle, guest editors checked the new version of the papers produced after the review to establish whether the authors carefully and adequately addressed the reviewers comments. When the reviewer 
comments were not completely addressed, a further cycle of review was required. Thus, for each peer-reviewed paper, the final decision was always confirmed by the guest editors. The papers were reviewed by a total of 17 reviewers. The names of the editorial committee members are listed on the acknowledgements of this editorial.

\section{Conclusion}

The Future Internet of Services and Things is becoming a reality with a plethora of software and hardware computing entities, such as services, sensors/actuators and other physical devices, bound to rapidly evolve. Providing verification and composition techniques and related tools to build innovative future applications, while dealing with the availability of a virtually infinite number of services and things, requires us to rethink service composition methods and verification techniques as devised for today's Internet. Thus, accounting for the overall service-oriented interaction pattern underlying the SOC paradigm, and for the IoT paradigm, future research directions should address crosscutting concerns on future systems verification and composition.

As pointed out by Brambilla et al. [3], the ultimate goal is to provide tangible value to the people. Thus, the need for novel approaches to automated composition that enables the connected services and things to flexibly work together, dynamically cooperate and securely exchange information goes together with the need for novel approaches to the realization of appropriate user interfaces, which permit to visualize information, let people navigate the information, and also interact with the systems, by dynamically setting composition properties and regulating the behavior of the resulting composite system.

Furthermore, as discussed by Bucchiarone et al. [4], the execution environment of service-oriented and thingbased modern applications is highly dynamic and, as a consequence, the service composition, and its verification against the application requirements, is considered to be a kind of every-minute routine activity. It has to be flexibly and quickly adapted to the rapidly changing environment. For instance, this is particularly true for user-centric systems, whose operation evolves around the needs and constraints of a specific user. In this case, the choice of services and composition objectives are determined by user's environment and personal preferences, constraints, or goals. Thus, it is quite clear that predefined, tailored and frozen solutions are not going to work in these IoS- and IoT-based systems. Indeed, each composition heavily depends on the execution context, e.g., the run-time parameters of the execution environment, the set of available services, the concrete user's needs, etc. This calls for dynamic composition frameworks that automate the whole service composition and verification life-cycle, from requirements specification and elicitation, to composition generation, to deployment and execution.

\section{Acknowledgements}

We thank all the authors, reviewers, editors-in-chief, and staff for the great work, which supported this thematic series on this very important topic both for the research community and for industry. In particular, we thank all the editorial committee members: Romain Rouvoy (University of Lille, FRANCE), Paulo Ferreira (University of Lisbon, PORTUGAL), Mauro Caporuscio (Linne University, SWEDEN), Grace Lewis (Carnegie Mellon University, UNITED STATES), Edoardo Patti (Politecnico di Torino, ITALY), Ella Pereira (Edge Hill University, UNITED KINGDOM), Federico Ciccozzi (Malardalen University, SWEDEN), Ludovico lovino (Gran Sasso Science Institute, ITALY), Jesús Sánchez Cuadrado (Universidad de Murcia, SPAIN), Antonis Bikakis (University College London, UNITED KINGDOM), Nikolaos Georgantas (Inria, FRANCE), Romina Spalazzese (University of Malmo, SWEDEN), Pascal Poizat (University of Paris Nanterre, FRANCE), Michael Sheng (Macquarie University, AUSTRALIA), Lionel Seinturier (University of Lille, FRANCE), Feda AIShahwan (University of Kuwait, KUWAIT), Ahmet Soylu (Norwegian University of Science and Technology, NORWAY).

The voluntary work of these researchers and practitioners were crucial for this thematic series.

This Thematic Series has been supported by (i) the European Union's H2020

Programme under grant agreement number 644178 (project

CHOReVOLUTION - Automated Synthesis of Dynamic and Secured

Choreographies for the Future Internet), (ii) the Ministry of Economy and

Finance, Cipe resolution n. 135/2012 (project INCIPICT - INnovating Clty

Planning through Information and Communication Technologies), and (iii) the GAUSS national research project, which has been funded by the MIUR under the PRIN 2015 program (Contract 2015KWREMX).

\section{Authors' contributions}

All authors read and approved the final manuscript.

\section{Competing interests}

The authors declare that they have no competing interests.

\section{Publisher's Note}

Springer Nature remains neutral with regard to jurisdictional claims in published maps and institutional affiliations.

\section{Author details}

${ }^{1}$ Department of Information Engineering, Computer Science and Mathematics - University of L'Aquila, L'Aquila, Italy. ${ }^{2}$ NASA Ames Research Center, Moffett Field, CA, USA.

Received: 11 March 2018 Accepted: 11 March 2018

Published online: 17 April 2018

\section{References}

1. Tan L, Wang N. Future internet: The internet of things. In: 2010 3rd International Conference on Advanced Computer Theory and Engineering(ICACTE), vol. 5. 2010. p. 5-3765380. https://doi.org/10.1109/ ICACTE.2010.5579543.

2. European Commission. Future Internet Research and Experimentation (FIRE) initiative. 2018. https://www.ict-fire.eu.

3. Brambilla M, Umuhoza E, Acerbis R. Model-driven development of user interfaces for iot systems via domain-specific components and patterns. J Internet Serv Appl. 2017;8(1):14. https://doi.org/10.1186/s13174-0170064-1.

4. Bucchiarone A, Marconi A, Pistore M, Raik H. A context-aware framework for dynamic composition of process fragments in the internet of services. J Internet Serv Appl. 2017;8(1):6. https://doi.org/10.1186/s13174-0170057-0. 